\title{
Lie Completion of Pseudo-Groups
}

Vladimir Itskov

Department of Mathematics

University of Nebraska

Lincoln, NE 68588

U.S.A.

vitskov2@math.unl.edu

http://www . math. unl. edu/ vitskov2
Peter J. Olver ${ }^{1}$

School of Mathematics

University of Minnesota

Minneapolis, MN 55455

U.S.A.

olver@math.umn .edu

http://www. math. umn.edu/ olver

Francis Valiquette ${ }^{2}$

Department of Mathematics and Statistics

McGill University

Montréal, Québec H3A 2K6

Canada

valiquette@math.mcgill.ca

http://www.math.mcgill.ca/valiquette

\begin{abstract}
By far the most important class of pseudo-groups, both for theory and in essentially all applications, are the Lie pseudo-groups. In this paper, we propose a definition of the Lie completion of a regular pseudo-group, and establish some of its basic properties. In particular, a pseudo-group and its Lie completion have exactly the same differential invariants and invariant differential forms. Thus, for practical purposes, one can exclusively work within the category of Lie pseudo-groups.
\end{abstract}

\footnotetext{
${ }^{1}$ Supported in part by NSF Grant 08-07317.

${ }^{2}$ Supported by a University of Minnesota Graduate School Doctoral Dissertation Fellowship and NSF Grant 05-05293.
} 


\section{Introduction}

In Lie's seminal work on transformation groups, the finite- and infinite-dimensional cases were always treated on essentially the same footing. However, through subsequent research, the two theories came to evolve in radically different ways. In the early twentieth century, finite-dimensional actions were codified and thereby well understood through the general theory of Lie groups. In contrast, the theory of infinite-dimensional pseudo-groups remains in a surprisingly primitive state of development, being characterized by André Weil, [20], as an "almost impenetrable jungle". The crux of the difficulty stems from the fact that there is, to date, no geometric object that adequately represents the abstract pseudo-group, and so pseudo-groups remain wedded to their individual actions on geometric spaces. A second complication is the distinction between a general pseudo-group and the more important, but more restrictive concept of a Lie pseudo-group. The axioms for plain pseudo-groups are straightforwardly adapted from those of local transformation groups, while a Lie pseudogroup must be realized as the space of solutions to a suitable system of differential equations - its determining equations - a non-geometric requirement that serves to complicate the theoretical foundations of the subject. An important question, thus, is the precise role played by the analytic requirement that the pseudo-group transformations satisfy a system of differential equations.

The purpose of this note is to describe a procedure for "completing" a sufficiently regular pseudo-group into an essentially equivalent Lie pseudo-group, as first outlined in [5]. The Lie completion of a pseudo-group is characterized as the smallest Lie pseudo-group that contains it. The existence of the completion follows from a direct construction of the determining system of differential equations satisfied by the pseudo-group's transformations, and the Lie completion contains all the solutions to these determining equations. As an immediate consequence of the construction, the original pseudo-group and its Lie completion are found to possess exactly the same differential invariants and invariant differential forms. In summary, the main conclusion is that, as far as local geometry and analysis is concerned, one can, without any appreciable loss of generality, always assume that a (suitably regular) pseudogroup satisfies the additional conditions that it be a Lie pseudo-group.

\section{Pseudo-Groups}

Let $M$ be an $m$-dimensional manifold. Throughout this note we adopt the definition that a local diffeomorphism $\phi: U \rightarrow M$ is a differentiable map defined on an open set $U=\operatorname{dom} \phi \subset$ $M$, with the property that it is a diffeomorphism onto its image.

We now recall the basic definition of a pseudo-group, $[12,17]$. To avoid technical complications, we will be working in the analytic category. Adapting the constructions to smooth $\left(C^{\infty}\right)$ pseudo-groups requires some additional care, as noted below.

Definition 2.1. Let $M$ be an analytic manifold. A collection $\mathcal{G}$ of local analytic diffeomorphisms of $M$ is a pseudo-group if

1. $\mathcal{G}$ is closed under restriction: if $U \subset M$ is an open set and $\phi: U \rightarrow M$ is in $\mathcal{G}$, then so is $\phi \mid V$ for all open $V \subset U$. 
2. Elements of $\mathcal{G}$ can be pieced together: if $U_{\nu} \subset M$ are open subsets, $U=\bigcup_{\nu} U_{\nu}$, and $\phi: U \rightarrow M$ is a local diffeomorphism with $\phi \mid U_{\nu} \in \mathcal{G}$ for all $\nu$, then $\phi \in \mathcal{G}$.

3. $\mathcal{G}$ contains the identity diffeomorphism: $\mathbb{1}(z)=z$ for all $z \in M=\operatorname{dom} \mathbb{1}$.

4. $\mathcal{G}$ is closed under composition: if $\phi: U \rightarrow M$ and $\psi: V \rightarrow M$ are two diffeomorphisms belonging to $\mathcal{G}$, and $\phi(U) \subset V$, then $\psi \circ \phi \in \mathcal{G}$.

5. $\mathcal{G}$ is closed under inverse: if $\phi: U \rightarrow M$ is in $\mathcal{G}$, then $\phi^{-1}: \phi(U) \rightarrow M$ is also in $\mathcal{G}$.

Example 2.2. The collection $\mathcal{D}=\mathcal{D}(M)$ of local analytic diffeomorphisms of an analytic manifold $M$ is the simplest example of a pseudo-group. All others are sub-pseudo-groups of the diffeomorphism pseudo-group. Well studied examples include analytic (local) actions of finite-dimensional Lie groups; the pseudo-group of symplectomorphisms, that is, canonical transformations of a symplectic manifold, [9]; foliation-preserving transformation groups, [4]; and a wide range of symmetry pseudo-groups of differential equations, [10].

Example 2.3. The collection $\mathcal{G}_{z_{0}} \subset \mathcal{D}$ of local analytic diffeomorphisms of $M$ which fix a point $z_{0} \in M$, i.e., satisfy $\phi\left(z_{0}\right)=z_{0}$, is a pseudo-group. More generally, if $S \subset M$ is any subset, its isotropy subgroup $\mathcal{G}_{S}=\{\phi \in \mathcal{D} \mid \phi(z)=z$ for all $z \in S\}$ forms a pseudo-group.

Example 2.4. The diagonal action of the one-dimensional diffeomorphism pseudo-group $\mathcal{D}(\mathbb{R})$ on $\mathbb{R}^{2}$ consists of all transformations of the form

$$
X=f(x), \quad Y=f(y)
$$

in which $f \in \mathcal{D}(\mathbb{R})$ is a local analytic diffeomorphism. It can be straightforwardly checked that this defines a pseudo-group, which, for later reference, we denote by $\mathcal{G}_{d}$.

As first emphasized by Ehresmann, [3], the analysis of pseudo-groups rests on the groupoid formed by their jets. The ur-example is the diffeomorphism pseudo-group $\mathcal{D}=\mathcal{D}(M)$. For each $0 \leq n \leq \infty$, let $\mathcal{D}^{(n)} \subset \mathrm{J}^{n}=\mathrm{J}^{n}(M, M)$ denote the bundle formed by the $n^{\text {th }}$ order jets, which, by the Inverse Function Theorem, is characterized by the non-vanishing of the Jacobian determinant. The $n^{\text {th }}$ order jet of a local diffemorphism $\phi \in \mathcal{D}$ is denoted by $\mathrm{j}_{n} \phi \subset$ $\mathcal{D}^{(n)}$. For $k \leq n$, let $\pi_{k}^{n}: \mathcal{D}^{(n)} \rightarrow \mathcal{D}^{(k)}$ denote the standard projection. The diffeomorphism jet bundle $\mathcal{D}^{(n)}$ carries the structure of a Lie groupoid, [8], whose multiplication is provided by algebraic composition of Taylor series (when defined).

Local coordinates $\left(z, Z^{(n)}\right)$ on $\mathcal{D}^{(n)}$ are provided by a system of source coordinates $z=$ $\left(z^{1}, \ldots, z^{m}\right)$ on $M$, target coordinates $Z=\left(Z^{1}, \ldots, Z^{m}\right)$ also on $M$, and jet coordinates $Z_{A}^{b}$ representing the partial derivatives $\partial^{k} \phi^{b}(z) / \partial z^{a_{1}} \cdots \partial z^{a_{k}}$, with $1 \leq b, a_{1}, \ldots, a_{k} \leq m$, $1 \leq k \leq n$, of the local diffeomorphism $Z=\phi(z)$. In what follows, we will consistently follow Cartan's convention to employ lower case letters, $z, x, u, \ldots$, for the source coordinates and upper case letters, $Z, X, U, \ldots$, for the corresponding target coordinates of a pseudo-group transformation. The source and target projections $\sigma, \tau: \mathcal{D}^{(n)} \rightarrow M$ are given by $\sigma\left(z, Z^{(n)}\right)=z$ and $\tau\left(z, Z^{(n)}\right)=Z$, respectively.

Given a sub-pseudo-group $\mathcal{G} \subset \mathcal{D}$, let $\mathcal{G}^{(n)} \subset \mathcal{D}^{(n)}$ denote the corresponding subgroupoid consisting of the $n^{\text {th }}$ order jets of its local diffeomorphisms. To avoid extra complications, we will impose the following regularity condition. 
Definition 2.5. A pseudo-group $\mathcal{G} \subset \mathcal{D}$ is called regular of order $n^{\star} \geq 1$ if, for all finite $n \geq n^{\star}$, the pseudo-group jets $\sigma: \mathcal{G}^{(n)} \rightarrow M$ form an embedded subbundle of $\sigma: \mathcal{D}^{(n)} \rightarrow M$, and, furthermore, the projection $\pi_{n}^{n+1}: \mathcal{G}^{(n+1)} \rightarrow \mathcal{G}^{(n)}$ is a fibration.

The isotropy pseudo-group $\mathcal{G}_{z_{0}}$ of Example 2.3 fails to be regular since its jet fibers over the point $z_{0}$ are of lower dimension, i.e. singular. Similarly the more general isotropy pseudogroup $G_{S}$ has singular fibers when $S \subset M$ is discrete. On the other hand, if the set $S$ has a non-empty interior, then its analytic isotropy pseudo-group just consists of the identity transformation, and so is trivially regular. (Although the corresponding smooth isotropy pseudo-group may be non-regular.) The diagonal pseudo-group $\mathcal{G}_{d}$ in Example 2.4 is also not regular since the jet fibers over the line $D=\{x=y\}$ are singular. On the other hand, $\mathcal{G}_{d}$ does act regularly on the complement $M=\mathbb{R}^{2} \backslash D$.

From here on, we will assume, by possibly discarding the subset belonging to the singular jet fibers, that all pseudo-group actions are regular.

\section{Lie Pseudo-Groups}

Lie pseudo-groups are distinguished by the additional property that their transformations form the space of solutions to a suitable system of differential equations. The literature contains several versions of the appropriate technical hypotheses, and we adopt a streamlined definition that accords with our restriction to the analytic category. The connections with more standard definitions, e.g. $[12,17]$, will be commented on below.

Definition 3.1. An analytic pseudo-group $\mathcal{G} \subset \mathcal{D}$ is called a Lie pseudo-group if $\mathcal{G}$ is regular of order $n^{\star} \geq 1$ and, moreover, every local diffeomorphism $\phi \in \mathcal{D}$ satisfying $\mathrm{j}_{n^{\star}} \phi \subset \mathcal{G}^{\left(n^{\star}\right)}$ belongs to the pseudo-group: $\phi \in \mathcal{G}$. The minimal value of $n^{\star}$ is called the order of the Lie pseudo-group.

The regularity condition implies that, in suitable local coordinates, the jet subbundle $\mathcal{G}^{\left(n^{\star}\right)} \subset \mathcal{D}^{\left(n^{\star}\right)}$ can be described by a system of $n^{\star \text { th }}$ order differential equations

$$
F^{\left(n^{\star}\right)}\left(z, Z^{\left(n^{\star}\right)}\right)=0,
$$

called the determining system of the pseudo-group ${ }^{1}$. The fact that $\mathcal{G}^{\left(n^{\star}\right)}$ is precisely the set of jets of pseudo-group elements implies that the determining system (3.1) is automatically locally solvable, [10], meaning that, for every jet $\left(z_{0}, Z_{0}^{\left(n^{\star}\right)}\right) \in \mathcal{G}^{\left(n^{\star}\right)}$, there exists a solution $Z=\phi(z)$ which has this jet at the point $z_{0}$, i.e., $\left.\mathrm{j}_{n^{\star}} \phi\right|_{z_{0}}=\left(z_{0}, Z_{0}^{\left(n^{\star}\right)}\right)$. The key condition that distinguishes a Lie pseudo-group is the requirement that every solution to the determining system belongs to the pseudo-group.

Example 3.2. Consider the diagonal pseudo-group $\mathcal{G}_{d}$ of Example 2.4, acting on the offdiagonal subset $M=\{(x, y) \mid x \neq y\}$ via $X=f(x), Y=f(y)$. Its jet subbundle $\mathcal{G}_{d}^{(1)} \subset \mathcal{D}^{(1)}$ is defined by the first order determining system

$$
X_{y}=Y_{x}=0,
$$

\footnotetext{
${ }^{1}$ Bear in mind that the regularity assumptions place additional regularity conditions on the nature of the determining partial differential equations.
} 
along with the inequalities $X_{x}, Y_{y} \neq 0$ guaranteeing that $\left(x, y, X, Y, X_{x}, X_{y}, Y_{x}, Y_{y}\right)$ is a local diffeomorphism jet. The higher order subbundles $\mathcal{G}_{d}^{(n)}$ are obtained by prolongation (differentiation) of the first order determining system (3.2); for example, $\mathcal{G}_{d}^{(2)} \subset \mathcal{D}^{(2)}$ is defined by the second order determining system

$$
X_{y}=Y_{x}=0, \quad X_{x y}=X_{y y}=Y_{x x}=Y_{x y}=0 .
$$

Even though it acts regularly away from the diagonal, $\mathcal{G}_{d}$ does not form a Lie pseudogroup. Indeed, the general solution to the determining system (3.2) (and its higher order counterparts) consists of all transformations of the form

$$
X=f(x), \quad Y=g(y),
$$

where $f, g \in \mathcal{D}(\mathbb{R})$ are arbitrary local diffeomorphisms. Clearly, (3.4) defines a larger pseudogroup, denoted $\overline{\mathcal{G}}_{d} \supsetneq \mathcal{G}_{d}$. By construction, $\overline{\mathcal{G}}_{d}$ has the same determining system: $\overline{\mathcal{G}}_{d}^{(n)}=\mathcal{G}_{d}^{(n)}$ for all $n$. Therefore, every solution to its determining system has the form (3.4). As a result, $\overline{\mathcal{G}}_{d}$ satisfies the conditions of Definition 3.1, and is a Lie pseudo-group. We will later identify $\overline{\mathcal{G}}_{d}$ as the Lie completion of the non-Lie pseudo-group $\mathcal{G}_{d}$.

In most treatments of the subject, the definition of a Lie pseudo-group imposes an additional integrability condition on its determining system. We will argue that, in the analytic category, the determining system is automatically integrable by construction. On the other hand, in the smooth category, the possibility of Lewy-type counterexamples to the required existence theorems, [10], appears to necessitate retaining an explicit integrability condition in the definition.

Recall that the $k^{\text {th }}$ prolongation of a system of differential equations is defined as the system obtained by appending all derivatives of the original equations of orders $\leq k,[1,16]$. We write $\mathrm{pr}^{(k)}$ for the prolongation operation.

Definition 3.3. Let $\Delta^{\left(n^{\star}\right)} \subset \mathrm{J}^{n^{\star}}$ be a system of differential equations of order $n^{\star}$ as in (3.1). For $n>n^{\star}$, we set $\Delta^{(n)}=\operatorname{pr}^{\left(n-n^{\star}\right)} \Delta^{\left(n^{\star}\right)} \subset \mathrm{J}^{n}$ to be the prolonged system of order $n$. Then $\Delta^{\left(n^{\star}\right)}$ is called formally integrable if $\pi_{n}^{n+1} \Delta^{(n+1)}=\Delta^{(n)}$ for all $n \geq n^{\star}$.

Loosely speaking, a system is formally integrable if every jet belonging to $\Delta^{\left(n^{\star}\right)}$ admits a formal power series solution of the infinitely prolonged system. This has the implication that the system does not admit integrability conditions stemming from cross differentiation of the equations, or, more precisely, by the processes of prolongation and jet projection. Involutivity is a more technical requirement, and, for brevity, we refer the reader to $[1,11,15,16]$ for the details. While formal integrability is more general, it cannot be checked by a finite algorithm, and only produces formal solutions. On the other hand, involutivity can be checked algorithmically, and, moreover, the Cartan-Kähler Theorem guarantees the existence of genuine solutions to an involutive analytic system.

Thanks to several fundamental results in the theory of overdetermined systems of partial differential equations, the integrability/involutivity of the determining system of an analytic Lie pseudo-group is, in fact, an immediate consequence of its regularity. The argument proceeds as follows. Let $\mathcal{G}$ be a Lie pseudo-group of order $n^{\star}$. By regularity and analyticity, the Cartan-Kuranishi Theorem, [7, 16], implies that some projection/prolongation $\widetilde{\mathcal{G}}^{(n)} \equiv$ 
$\pi_{n}^{k} \mathrm{pr}^{\left(k-n^{\star}\right)} \mathcal{G}^{\left(n^{\star}\right)} \subset \mathcal{D}^{(n)}, k \geq n \geq n^{\star}$, is involutive. We claim that $\widetilde{\mathcal{G}}^{(n)}=\mathcal{G}^{(n)}$. Indeed, any solution $\phi \in \mathcal{G}$ to the determining equations $\mathcal{G}^{\left(n^{\star}\right)}$ is automatically a solution to any prolongation and projection thereof, and hence satisfies the involutive system $\widetilde{\mathcal{G}}^{(n)}$. But $\mathcal{G}^{(n)}$ is, by definition, the set of all solution jets of order $n$, and thus $\mathcal{G}^{(n)} \subset \widetilde{\mathcal{G}}^{(n)}$. On the other hand, any solution to $\widetilde{\mathcal{G}}^{(n)}$ is necessarily a solution to the original system $\mathcal{G}^{\left(n^{\star}\right)}$ and thus, since $\mathcal{G}$ is, by assumption, a Lie pseudo-group, an element of $\mathcal{G}$. We conclude that $\widetilde{\mathcal{G}}^{(n)} \subset \mathcal{G}^{(n)}$, which establishes our claim. We have thus proved the following result, that justifies removing any integrability requirement from our definition of a (regular) analytic Lie pseudo-group.

Theorem 3.4. The determining equations of a regular analytic Lie pseudo-group of order $n^{\star}$ are necessarily formally integrable and, in fact, involutive at some order $n \geq n^{\star}$.

In contrast, there are no comparable existence theorems for formally integrable or involutive systems of partial differential equations in the smooth category. Indeed, it is conceivable that a $C^{\infty}$ system of partial differential equations be locally solvable and yet not formally integrable due to some "hidden integrability condition" satisfied by its higher order jets that cannot be deduced by prolongation. In more detail, it may be possible that a smooth pseudo-group $\mathcal{G}$ satisfy the conditions of Definition 3.1, and yet, for some $n>n^{\star}$, the $n^{\text {th }}$ order pseudo-group jets form a strict subbundle of the prolonged determining system: $\mathcal{G}^{(n)} \subsetneq \mathrm{pr}^{\left(n-n^{\star}\right)} \mathcal{G}^{\left(n^{\star}\right)}$. In other words, some of the differential equations required to specify $\mathcal{G}^{(n)}$ do not result from differentiating its determining system. Finding such an example or, alternatively, proving that such does not exist — is a challenging problem.

\section{Completion}

We are now in a position to present our definition of the Lie completion of a regular pseudogroup action and prove the main result.

Definition 4.1. Let $\mathcal{G}$ be a regular pseudo-group of order $n^{\star}$. Then its Lie completion $\overline{\mathcal{G}} \supset \mathcal{G}$ is defined as the space of all analytic solutions $\phi$ of the determining system $\mathcal{G}^{\left(n^{\star}\right)}$ that determine one-to-one maps on their domain of definition.

Since $\mathcal{G}^{\left(n^{\star}\right)} \subset \mathcal{D}^{\left(n^{\star}\right)}$, the Inverse Function Theorem implies that any solution $\phi$ to $\mathcal{G}^{\left(n^{\star}\right)}$ is a local diffeomorphism when restricted to a sufficiently small open subset of its domain. Thus, all solutions to $\mathcal{G}^{\left(n^{\star}\right)}$ are one-to-one maps on suitable open subsets of their domains of definition, and these restrictions will all belong to the completion $\overline{\mathcal{G}}$. While an immediate consequence of Definition 4.1, the next lemma is fundamental for proving the main properties of Lie completion.

Lemma 4.2. Let $\mathcal{G}$ be a regular pseudo-group of order $n^{\star}$ and $n \geq n^{\star}$. Then for any element $\bar{\phi} \in \overline{\mathcal{G}}$ of the completion and $z \in \operatorname{dom} \bar{\phi}$ a point in its domain there exists a pseudo-group element $\phi \in \mathcal{G}$ (depending on both $z$ and $n$ ) that has the same $n^{\text {th }}$ order jet: $\left.\mathrm{j}_{n} \bar{\phi}\right|_{z}=\left.\mathrm{j}_{n} \phi\right|_{z}$.

In simpler terms, the Taylor polynomial of degree $n$ of any element of the completion is also the Taylor polynomial of an element of the original pseudo-group. Note that this holds at arbitrary finite order $n \geq n^{\star}$, but not necessarily at order $n=\infty$ - the level of Taylor series. 
Theorem 4.3. The Lie completion $\overline{\mathcal{G}}$ of a regular pseudo-group $\mathcal{G}$ is a Lie pseudo-group. Moreover, if $\mathcal{G}$ is itself a Lie pseudo-group then $\overline{\mathcal{G}}=\mathcal{G}$.

Proof. We begin by showing that $\overline{\mathcal{G}}$ is indeed a pseudo-group. To verify the pseudo-group axioms in Definition 2.1 for $\overline{\mathcal{G}}$, we first note that the restriction and piecing together properties are immediate. Since the identity map belongs to $\mathcal{G}$, it automatically belongs to $\overline{\mathcal{G}}$. Next, to verify closure of $\overline{\mathcal{G}}$ under composition, suppose $\bar{\phi}, \bar{\psi} \in \overline{\mathcal{G}}$. Applying Lemma 4.2, given $z \in \operatorname{dom} \bar{\phi}$ such that $\bar{\phi}(z) \in \operatorname{dom} \bar{\psi}$, choose $\phi, \psi \in \mathcal{G}$ (depending on the point $z$ and the order $\left.n \geq n^{\star}\right)$ such that

$$
\left.\mathrm{j}_{n} \bar{\phi}\right|_{z}=\left.\mathrm{j}_{n} \phi\right|_{z},\left.\quad \mathrm{j}_{n} \bar{\psi}\right|_{\bar{\phi}(z)}=\left.\mathrm{j}_{n} \psi\right|_{\phi(z)},
$$

noting that $\bar{\phi}(z)=\phi(z)$ thanks to the first equality. Because $\mathcal{G}$ is closed under composition,

$$
\left.\mathrm{j}_{n}(\bar{\psi} \circ \bar{\phi})\right|_{z}=\left.\left.\mathrm{j}_{n} \bar{\psi}\right|_{\bar{\phi}(z)} \circ \mathrm{j}_{n} \bar{\phi}\right|_{z}=\left.\left.\mathrm{j}_{n} \psi\right|_{\phi(z)} \circ \mathrm{j}_{n} \phi\right|_{z}=\left.\left.\mathrm{j}_{n}(\psi \circ \phi)\right|_{z} \in \mathcal{G}^{(n)}\right|_{z} .
$$

Since the point $z$ is arbitrary, setting $n=n^{\star}$, we deduce that $\bar{\psi} \circ \bar{\phi}$ is a solution to the determining system $\mathcal{G}^{\left(n^{\star}\right)}$, and hence belongs to $\overline{\mathcal{G}}$. A similar argument proves closure under inversion. Let $\bar{\phi} \in \overline{\mathcal{G}}$, and, for $z \in \operatorname{dom} \bar{\phi}$, choose $\phi \in \mathcal{G}$ satisfying the first condition in (4.1). Noting that

$$
\left.\left.\mathrm{j}_{n} \bar{\phi}^{-1}\right|_{\bar{\phi}(z)} \circ \mathrm{j}_{n} \bar{\phi}\right|_{z}=\left.\mathrm{j}_{n} \mathbb{1}\right|_{z}=\left.\left.\mathrm{j}_{n} \phi^{-1}\right|_{\phi(z)} \circ \mathrm{j}_{n} \phi\right|_{z},
$$

we must have $\left.\mathrm{j}_{n} \bar{\phi}^{-1}\right|_{\bar{\phi}(z)}=\left.\left.\mathrm{j}_{n} \phi^{-1}\right|_{\phi(z)} \in \mathcal{G}^{(n)}\right|_{\phi(z)}$. Again, as $z$ is arbitrary, for $n=n^{\star}$, this implies that $\bar{\phi}^{-1}$ is a solution to the determining system $\mathcal{G}^{\left(n^{\star}\right)}$, and hence belongs to $\overline{\mathcal{G}}$. We conclude that $\overline{\mathcal{G}}$ is indeed a pseudo-group.

Obviously, by construction, every order $n^{\star}$ jet of a diffeomorphism in $\overline{\mathcal{G}}$ coincides with the $n^{\star}$ jet of some diffeomorphism in $\mathcal{G}$, so $\overline{\mathcal{G}}^{\left(n^{\star}\right)}=\mathcal{G}^{\left(n^{\star}\right)}$. Moreover, every solution to the determining system $\overline{\mathcal{G}}^{\left(n^{\star}\right)}=\mathcal{G}^{\left(n^{\star}\right)}$ belongs to $\overline{\mathcal{G}}$, and hence $\overline{\mathcal{G}}$ is indeed a Lie pseudo-group. The final statement of the theorem is an immediate consequence of the definition of Lie completion.

There is another natural way of defining the completion of a pseudo-group based on the partial ordering provided by inclusion of pseudo-groups.

Theorem 4.4. The Lie completion $\overline{\mathcal{G}}$ of a regular pseudo-group $\mathcal{G} \subset \mathcal{D}$ of order $n^{\star}$ is the smallest Lie pseudo-group satisfying $\mathcal{G} \subset \overline{\mathcal{G}} \subset \mathcal{D}$. In other words,

$$
\overline{\mathcal{G}}=\bigcap_{\mathcal{H} \supset \mathcal{G}} \mathcal{H}
$$

where the intersection is taken over all Lie pseudo-groups $\mathcal{G} \subset \mathcal{H} \subset \mathcal{D}$ of order $n \leq n^{\star}$.

Proof. Let $\mathcal{I}$ denote the right hand side of (4.3). Since the Lie completion $\overline{\mathcal{G}} \supset \mathcal{G}$ is a Lie pseudo-group, it is one of the $\mathcal{H}$ 's appearing in the intersection $\mathcal{I}$. Thus, trivially, $\mathcal{I} \subset \overline{\mathcal{G}}$.

On the other hand, suppose $\mathcal{H} \supset \mathcal{G}$ is a Lie pseudo-group of order $n \leq n^{\star}$. Our task is to prove that, given any $\bar{\phi} \in \overline{\mathcal{G}}$, then $\bar{\phi} \in \mathcal{H}$. Indeed, if this is true, then $\overline{\mathcal{G}} \subset \mathcal{H}$. Since this holds for every $\mathcal{H}$ appearing in (4.3), the same holds for the intersection: $\overline{\mathcal{G}} \subset \mathcal{I}$, which serves to complete the proof. 
To establish the preceding claim, we apply Lemma 4.2: for each $z \in \operatorname{dom} \bar{\phi}$ there exists a $\phi \in \mathcal{G}$ such that

$$
\left.\mathrm{j}_{n} \bar{\phi}\right|_{z}=\left.\left.\mathrm{j}_{n} \phi\right|_{z} \in \mathcal{H}^{(n)}\right|_{z} \quad \text { since } \phi \in \mathcal{G} \subset \mathcal{H} \text {. }
$$

(Keep in mind that $\phi$ will depend on $z$ as well as the jet $\left.j_{n} \bar{\phi}\right|_{z}$.) Since this holds for all $z \in \operatorname{dom} \bar{\phi}$, we conclude that $\bar{\phi}$ is a solution to the determining equations for $\mathcal{H}$. But $\mathcal{H}$ is, by assumption, a Lie pseudo-group, and hence $\bar{\phi} \in \mathcal{H}$, proving the result.

Example 4.5. A simple but revealing example is provided by the pseudo-group $\mathcal{G}_{\delta}$ acting on $\mathbb{R}^{4}$ whose transformations have the form

$$
X=f(x), \quad Y=f(y), \quad U=f^{\prime}(x) u, \quad V=f^{\prime}(y) v,
$$

for arbitrary $f \in \mathcal{D}(\mathbb{R})$. Observe that $\mathcal{G}_{\delta}$ acts regularly on

$$
M=\{(x, y, u, v) \mid x \neq y, u \neq 0, v \neq 0\} \subset \mathbb{R}^{4} .
$$

The first order determining equations of $\mathcal{G}_{\delta}$ are

$$
X_{y}=X_{u}=X_{v}=0, \quad Y_{x}=Y_{u}=Y_{v}=0, \quad U=X_{x} u, \quad V=Y_{y} v .
$$

The higher order determining equations are obtained by prolongation.

By definition, the Lie completion of $\mathcal{G}_{\delta}$ is the solution space $\overline{\mathcal{G}}_{\delta}$ to the determining system (4.5), which is the set of diffeomorphisms of the form

$$
X=f(x), \quad Y=g(y), \quad U=f^{\prime}(x) u, \quad V=g^{\prime}(y) v,
$$

for $f, g \in \mathcal{D}(\mathbb{R})$. Theorem 4.3 assures us that $\overline{\mathcal{G}}_{\delta}$ is a Lie pseudo-group acting on $M$.

On the other hand, we can clearly extend the action (4.6) to all of $\mathbb{R}^{4}$, but the resulting pseudo-group is not regular. Indeed, the jet fibers sitting over the subset $\{u=v=0\}$ are singular, and hence the extension does not satisfy our criteria for a Lie pseudo-group.

This example points out the importance of the regularity conditions for the definition of the Lie completion of a pseudo-group. Further investigation indicates that this appears to depend upon whether any source coordinates are present in the determining equations for the pseudo-group. In our example, the source coordinates $x$ and $y$ do not explicitly appear in the determining system (4.5). Indeed, $\overline{\mathcal{G}}_{\delta}$ can be extended to a Lie pseudo-group acting on $\mathbb{R}^{4} \backslash\{u=v=0\}$, but not all of $\mathbb{R}^{4}$.

We next show that the Lie completion of a pseudo-group is unique up to isomorphism. For this purpose, we recall Cartan's and Vessiot's notion of isomorphism in the category of regular pseudo-group actions, [2, 19].

Definition 4.6. Let $\mathcal{G}$ be a regular pseudo-group action. Its $n^{\text {th }}$ order normal prolongation is the induced action of $\mathcal{G}$ on $\mathcal{G}^{(n)}$.

Example 4.7. The first order normal prolongation of the pseudo-group action (2.1) is the pseudo-group (4.4). 
Definition 4.8. Two regular pseudo-group actions $\mathcal{G}_{1}, \mathcal{G}_{2}$ on manifolds $M_{1}, M_{2}$ are isomorphic, written $\mathcal{G}_{1} \sim \mathcal{G}_{2}$, if they have a common isomorphic normal prolongation, meaning a regular pseudo-group $\mathcal{G}$ acting on a manifold $M$, and surjective submersions $\pi_{i}: M \rightarrow M_{i}$, $i=1,2$, such that, for each $i=1,2$, there is a one-to-one correspondence between elements $\phi \in \mathcal{G}$ and $\phi_{i} \in \mathcal{G}_{i}$ satisfying $\pi_{i} \circ \phi=\phi_{i} \circ \pi_{i}$.

Example 4.9. The pseudo-group (4.4) is isomorphic to the pseudo-group (2.1). To see this, let $M_{1}=\{(x, y) \mid x \neq y\}$ and $M=M_{2}=\{(x, y, u, v) \mid x \neq y, u \neq 0, v \neq 0\}$. Then the appropriate surjective submersions are given by $\pi_{1}(x, y, u, v)=(x, y)$ and $\pi_{2}=\mathbb{1}$.

Example 4.10. In terms of Definition 4.8, the pseudo-group (2.1) is not isomorphic to $\mathcal{D}\left(\mathbb{R}^{1}\right)$.

Theorem 4.11. Let $\mathcal{G}_{1} \sim \mathcal{G}_{2}$ be two isomorphic regular pseudo-groups with respective Lie completions $\overline{\mathcal{G}}_{1}, \overline{\mathcal{G}}_{2}$. Then $\overline{\mathcal{G}}_{1} \sim \overline{\mathcal{G}}_{2}$.

Proof. Let $\mathcal{G}$ be an isomorphic normal prolongation of the regular pseudo-groups $\mathcal{G}_{1}, \mathcal{G}_{2}$. Let $n_{1}^{\star}, n_{2}^{\star}, n^{\star}$ be the orders of regularity of $\mathcal{G}_{1}, \mathcal{G}_{2}$ and $\mathcal{G}$, respectively, and define $\bar{n}=$ $\max \left\{n_{1}^{\star}, n_{2}^{\star}, n^{\star}\right\}$ to be the maximum of the three values. Let $\overline{\mathcal{G}}_{1}, \overline{\mathcal{G}}_{2}$ and $\overline{\mathcal{G}}$ be the respective Lie completions of $\mathcal{G}_{1}, \mathcal{G}_{2}$ and $\mathcal{G}$. To show $\overline{\mathcal{G}}_{1} \sim \overline{\mathcal{G}}_{2}$, we first note that, for each $i=1$, 2 , the equation $\pi_{i} \circ \phi=\phi_{i} \circ \pi_{i}$ reduces to

$$
\left.\left.\mathrm{j}_{n} \pi_{i}\right|_{\phi(z)} \circ \mathrm{j}_{n} \phi\right|_{z}=\left.\left.\mathrm{j}_{n} \phi_{i}\right|_{\pi_{i}(z)} \circ \mathrm{j}_{n} \pi_{i}\right|_{z},
$$

at the level of jets. Now choose $\bar{\phi} \in \overline{\mathcal{G}}$ and $z \in \operatorname{dom} \bar{\phi}$. Then, for each $n \geq \bar{n}$, equation (4.7) and Lemma 4.2 imply that there exists $\phi_{n} \in \mathcal{G}$ and corresponding $\phi_{n, i} \in \mathcal{G}_{i}$ such that

$$
\left.\left.\mathrm{j}_{n} \pi_{i}\right|_{\bar{\phi}(z)} \circ \mathrm{j}_{n} \bar{\phi}\right|_{z}=\left.\left.\mathrm{j}_{n} \pi_{i}\right|_{\phi_{n}(z)} \circ \mathrm{j}_{n} \phi_{n}\right|_{z}=\left.\left.\mathrm{j}_{n} \phi_{n, i}\right|_{\pi_{i}(z)} \circ \mathrm{j}_{n} \pi_{i}\right|_{z} .
$$

Taking the limit of (4.8) as $n \rightarrow \infty$, and denoting the limiting infinite jet by $\left.\mathrm{j}_{\infty} \bar{\phi}_{i}\right|_{\pi_{i}(z)}$, we obtain

$$
\left.\left.\mathrm{j}_{\infty} \pi_{i}\right|_{\bar{\phi}(z)} \circ \mathrm{j}_{\infty} \bar{\phi}\right|_{z}=\left.\left.\mathrm{j}_{\infty} \bar{\phi}_{i}\right|_{\pi_{i}(z)} \circ \mathrm{j}_{\infty} \pi_{i}\right|_{z}
$$

It is easy to see that $\left.\mathrm{j}_{\infty} \bar{\phi}_{i}\right|_{\pi_{i}(z)}$ is the infinite jet of a local analytic diffeomorphism $\bar{\phi}_{i}$ defined in some neighborhood $U_{i} \subset M_{i}$ of $\pi_{i}(z)$. By analyticity, the map $\bar{\phi}_{i}$ is uniquely determined as it is completely prescribed by its infinite jet at $\pi_{i}(z)$. Furthermore, since $\left.\left.\mathrm{j}_{\infty} \bar{\phi}_{i}\right|_{\pi_{i}(z)} \in \overline{\mathcal{G}}_{i}^{(\infty)}\right|_{z}$, the map $\bar{\phi}_{i}$ is in the Lie pseudo-group $\overline{\mathcal{G}}_{i}$. By construction, it also satisfies the identity $\pi_{i} \circ \bar{\phi}=\bar{\phi}_{i} \circ \pi_{i}$ on $\pi^{-1}\left(U_{i}\right) \cap \operatorname{dom} \bar{\phi}$. Next, we note that if $\bar{\phi} \neq \bar{\psi}$ then $\bar{\phi}_{i} \neq \bar{\psi}_{i}$. Indeed, choosing $z \in \operatorname{dom} \bar{\phi} \cap \operatorname{dom} \bar{\psi}$ such that there exists $n \geq \bar{n}$ satisfying $\left.\mathrm{j}_{n} \bar{\phi}\right|_{z} \neq\left.\mathrm{j}_{n} \bar{\psi}\right|_{z}$ we observe from (4.8) that the sequence defining the infinite jets of $\bar{\phi}_{i}$ and $\bar{\psi}_{i}$ must differ.

Similar argument, this time starting with $\bar{\phi}_{i} \in \overline{\mathcal{G}}_{i}$ to construct $\bar{\phi} \in \overline{\mathcal{G}}$, shows that the correspondence between the elements $\bar{\phi} \in \overline{\mathcal{G}}$ and $\bar{\phi}_{i} \in \overline{\mathcal{G}}_{i}$ is one-to-one. Here the assumption that $\mathcal{G}$ is an isomorphic prolongation of $\mathcal{G}_{i}$ is necessary to argue that $\bar{\phi}$ is analytic. This shows that $\overline{\mathcal{G}}$ is an isomorphic prolongation of $\overline{\mathcal{G}}_{i}$, and by a theorem of Cartan, [18], $\overline{\mathcal{G}}$ is isomorphic to some normal prolongation of $\overline{\mathcal{G}}_{i}$. 


\section{$5 \quad$ Differential Invariants}

In many applications, the principal object of study is the induced action of a pseudo-group on submanifolds. For instance, if the pseudo-group arises as the symmetry group of a system of differential equations, the submanifolds are the graphs of candidate solutions.

Let $\mathcal{G}$ be a pseudo-group acting on an $m$-dimensional manifold $M$. Fixing $1 \leq p<m$, we consider the induced action of $\mathcal{G}$ on $p$-dimensional submanifolds $S \subset M$. For $0 \leq n \leq \infty$, let $\mathrm{J}^{n}(M, p)$ denote the $n^{\text {th }}$ order (extended) jet bundle consisting of equivalence classes of $p$-dimensional submanifolds under the equivalence relation of $n^{\text {th }}$ order contact, [10]. In an adapted system of coordinates $z=\left(x^{1}, \ldots, x^{p}, u^{1}, \ldots, u^{q}\right)$, so that a submanifold $S$ is locally represented as the graph of a function $(x, u(x))$, the submanifold jets are denoted by $\left.\mathrm{j}_{n} S\right|_{z}=z^{(n)}=\left(x, u^{(n)}\right)$. Here, $u^{(n)}$ denotes the collection of derivatives of the dependent variables $u^{\alpha}$ with respect to the independent variables $x^{i}$ of order $0 \leq k \leq n$.

Since diffeomorphisms preserve contact, there is an induced action of $\mathcal{G}$ on the submanifold jet space $\mathrm{J}^{n}(M, p)$, known as the $n^{\text {th }}$ order prolonged ${ }^{2}$ action. For $z^{(n)}=\left.\mathrm{j}_{n} S\right|_{z} \in \mathrm{J}^{n}(M, p)$ and $\phi \in \mathcal{G}$ the prolonged action is defined by $\phi^{(n)}\left(z^{(n)}\right)=\left.\left.\mathrm{j}_{n} \phi\right|_{z} \cdot \mathrm{j}_{n} S\right|_{z}:=\left.\mathrm{j}_{n} \phi(S)\right|_{\phi(z)}$. In a local coordinate system, the prolonged action is obtained by implementing the standard chain rule, [13].

Definition 5.1. A differential invariant of a pseudo-group $\mathcal{G}$ is a function $I: W \rightarrow \mathbb{R}$, defined on an open subset $W \subset \mathrm{J}^{n}(M, p)$, that is unaffected by the prolonged pseudo-group action:

$$
I\left(\phi^{(n)}\left(z^{(n)}\right)\right)=I\left(z^{(n)}\right),
$$

for all $\phi \in \mathcal{G}$ and all submanifold jets $z^{(n)}$ such that both $z^{(n)}, \phi^{(n)}\left(z^{(n)}\right) \in W$.

Theorem 5.2. Let $\mathcal{G} \subset \mathcal{D}$ be a regular pseudo-group and $\overline{\mathcal{G}}$ its Lie completion. Then $\overline{\mathcal{G}}$ and $\mathcal{G}$ have the same differential invariants.

Proof. Since $\mathcal{G} \subset \overline{\mathcal{G}}$, any differential invariant of $\overline{\mathcal{G}}$ is automatically a differential invariant of $\mathcal{G}$. Conversely, suppose $I$ satisfies (5.1). Let $\left.z^{(n)} \in \mathrm{J}^{n}(M, p)\right|_{z}$. Given $\bar{\phi} \in \overline{\mathcal{G}}$ with $z \in \operatorname{dom} \bar{\phi}$, choose $\left.\phi \in \mathcal{G}\right|_{z}$ such that $\left.\mathrm{j}_{n} \bar{\phi}\right|_{z}=\left.\mathrm{j}_{n} \phi\right|_{z}$. By the chain rule, the prolonged action of a diffeomorphism $\phi$ on a jet $z^{(n)}$ depends only on its $n$ jet $\left.\mathrm{j}_{n} \phi\right|_{z}$ at the base point $z$. Therefore, $\bar{\phi}^{(n)}\left(z^{(n)}\right)=\phi^{(n)}\left(z^{(n)}\right)$, which, provided both $z^{(n)}$ and its image lie in the domain of definition of $I$, implies

$$
I\left(\bar{\phi}^{(n)}\left(z^{(n)}\right)\right)=I\left(\phi^{(n)}\left(z^{(n)}\right)\right)=I\left(z^{(n)}\right) .
$$

We conclude that $I\left(z^{(n)}\right)$ is also a differential invariant of $\overline{\mathcal{G}}$.

Example 5.3. Consider the non-Lie pseudo-group $\mathcal{G}$ acting on $\mathbb{R}^{3}$ via

$$
X=f(x), \quad Y=f(y), \quad U=u, \quad \text { where } \quad f \in \mathcal{D}(\mathbb{R}) .
$$

The action is regular on the open dense subset $M=\{(x, y, u) \mid x \neq y\}$, where the determining equations are provided by

$$
X_{y}=Y_{x}=U_{x}=U_{y}=0, \quad U_{u}=1, \quad X_{x}, Y_{y} \neq 0,
$$

\footnotetext{
${ }^{2}$ Not to be confused with the prolongation of differential systems defined above.
} 
along with their prolongations. The completion $\overline{\mathcal{G}}$ of this pseudo-group is the Lie pseudogroup action on $M$ given by

$$
X=f(x), \quad Y=g(y), \quad U=u, \quad f, g \in \mathcal{D}(\mathbb{R}) .
$$

We consider the induced action of these pseudo-groups on surfaces $S \subset M$. Restricting our attention to graphs of functions $u=h(x, y)$, the induced coordinates on the surface jet bundles $\mathrm{J}^{n}(M, 2)$ are denoted by $x, y, u, u_{x}, u_{y}, u_{x x}, u_{x y}, \ldots$. We use implicit differentiation to determine the prolonged actions, which, up to order 2 , are, respectively,

$$
\begin{aligned}
& X=f(x), \quad Y=f(y), \quad U=u, \quad U_{X}=\frac{u_{x}}{f^{\prime}(x)}, \quad U_{Y}=\frac{u_{y}}{f^{\prime}(y)}, \\
& \mathcal{G}: \quad U_{X X}=\frac{u_{x x} f^{\prime}(x)-u_{x} f^{\prime \prime}(x)}{f^{\prime}(x)^{3}}, \quad U_{X Y}=\frac{u_{x y}}{f^{\prime}(x) f^{\prime}(y)}, \quad U_{Y Y}=\frac{u_{y y} f^{\prime}(y)-u_{y} f^{\prime \prime}(y)}{f^{\prime}(y)^{3}}, \\
& \quad U^{3}=f(x), \quad Y=g(y), \quad U=u, \quad U_{X}=\frac{u_{x}}{f^{\prime}(x)}, \quad U_{Y}=\frac{u_{y}}{g^{\prime}(y)}, \\
& \overline{\mathcal{G}}: \quad U_{X X}=\frac{u_{x x} f^{\prime}(x)-u_{x} f^{\prime \prime}(x)}{f^{\prime}(x)^{3}}, \quad U_{X Y}=\frac{u_{x y}}{f^{\prime}(x) g^{\prime}(y)}, \quad U_{Y Y}=\frac{u_{y y} g^{\prime}(y)-u_{y} g^{\prime \prime}(y)}{g^{\prime}(y)^{3}} .
\end{aligned}
$$

In the case of $\overline{\mathcal{G}}$, since the values of $f^{\prime}(x), f^{\prime \prime}(x), g^{\prime}(y), g^{\prime \prime}(y)$ are independent of each other, there is (up to functions thereof) just one second order differential invariant, namely

$$
I=\frac{u_{x y}}{u_{x} u_{y}}
$$

On the other hand, for the non-Lie pseudo-group $\mathcal{G}$, when $x \neq y$, the values of $f$ and its derivatives at the point $x$ can be specified independently of the values at the point $y$. This implies that the prolonged action of $\mathcal{G}$ has the same second order differential invariant (5.4), in accordance with Theorem 5.2. Further, both pseudo-groups possess the same higher order differential invariants, which can all be found through repeated application of the invariant differentiation operators $\mathcal{D}_{1}=u_{x}^{-1} D_{x}, \mathcal{D}_{2}=u_{y}^{-1} D_{x}$. We note that the differential invariant $I$, the invariant differential operators $\mathcal{D}_{1}, \mathcal{D}_{2}$, and the full structure of the differential invariant algebra can all be systematically found by applying the method of moving frames for pseudogroups developed in $[13,14]$.

Invariant differential forms play an important role in the theory and applications of the method of moving frames, [13]. A similar argument shows that regular pseudo-groups have the same invariant differential forms as their Lie completion.

Definition 5.4. An invariant differential form of a pseudo-group $\mathcal{G}$ is a locally defined differential form $\Omega$ on the submanifold jet bundle $\mathrm{J}^{n}(M, p)$ that is unaffected by the pullback of the prolonged pseudo-group action:

$$
\left(\phi^{(n)}\right)^{*} \Omega=\Omega
$$

for all $\phi \in \mathcal{G}$ such that the domain and target of $\phi$ are contained in the domain of definition of $\Omega$. 
Using the same method of proof as in Theorem 5.2, we readily establish the general result.

Theorem 5.5. Let $\mathcal{G} \subset \mathcal{D}$ be a regular pseudo-group and $\overline{\mathcal{G}}$ its Lie completion. Then $\overline{\mathcal{G}}$ and $\mathcal{G}$ have the same invariant differential forms on $\mathrm{J}^{n}(M, p)$.

Thus, from the point of view of the local geometry of submanifolds, we can work with the Lie completion of a (regular) pseudo-group without any loss of information. Both possess the same system of differential invariants, invariant differential forms, and, as a consequence, the same invariant differential equations and variational principles. In other words, under the assumption of regularity, one can exclusively work with Lie pseudo-groups. On the other hand, extending these results to non-regular examples is worthy of further investigation.

Finally, we remark that H.H. Johnson, [6], introduced a rather different concept of a "complete Lie pseudo-group". Johnson's notion of completeness requires the existence of a finite system of nontrivial differential invariants that uniquely characterizes the infinitesimal generators of the pseudo-group (in a neighborhood of a point). This would leave out many examples, particularly those that have no differential invariants, but are nevertheless complete in our terminology, e.g. diffeomorphisms, symplectomorphisms, the completion $\overline{\mathcal{G}}_{d}$ of the diagonal pseudo-group of Example 3.2, and so on.

Acknowledgments: We would like to thank Juha Pohjanpelto and the anonymous referees for helpful remarks that served to improve the paper.

\section{References}

[1] Bryant, R.L., Chern, S.-S., Gardner, R.B., Goldschmidt, H.L., and Griffiths, P.A., Exterior Differential Systems, Math. Sci. Res. Inst. Publ., vol. 18, Springer-Verlag, New York, 1991.

[2] Cartan, É., Sur la structure des groupes infinis de transformations, in Oeuvres Complètes, Part. II, Vol. 2, Gauthier-Villars, Paris, 1953, pp. 571-714.

[3] Ehresmann, C., Introduction à la théorie des structures infinitésimales et des pseudogroupes de Lie, in: Géometrie Différentielle, Colloq. Inter. du Centre Nat. de la Rech. Sci., Strasbourg, 1953, pp. 97-110.

[4] Fuchs, D.B., Gabrielov, A.M., and Gel'fand, I.M., The Gauss-Bonnet theorem and Atiyah-Patodi-Singer functionals for the characteristic classes of foliations, Topology 15 (1976) 165-188.

[5] Itskov, V., Orbit Reduction of Exterior Differential Systems, Ph.D. Thesis, University of Minnesota, 2002.

[6] Johnson, H.H., Classical differential invariants and applications to partial differential equations, Math. Ann. 148 (1962) 308-329.

[7] Kuranishi, M., On the local theory of continuous infinite pseudo groups I, II, Nagoya Math. J. 15 (1959) 225-260, 19 (1961) 55-91. 
[8] Mackenzie, K., Lie Groupoids and Lie Algebroids in Differential Geometry, London Math. Soc. Lecture Notes, vol. 124, Cambridge University Press, Cambridge, 1987.

[9] McDuff, D., and Salamon, D., Introduction to Symplectic Topology, Oxford University Press, Oxford, 1995.

[10] Olver, P.J., Applications of Lie Groups to Differential Equations, Graduate Texts in Mathematics, vol. 107, Springer, New York, 1993.

[11] Olver, P.J., Equivalence, Invariants, and Symmetry, Cambridge University Press, Cambridge, 1995.

[12] Olver, P.J., and Pohjanpelto, J., Maurer-Cartan equations and structure of Lie pseudogroups, Selecta Math. 11 (2005) 99-126.

[13] Olver, P.J., and Pohjanpelto, J., Moving frames for Lie pseudo-groups, Canadian J. Math. 60 (2008) 1336-1386.

[14] Olver, P.J., and Pohjanpelto, J., Differential invariant algebras of Lie pseudo-groups, Adv. Math. 222 (2009) 1746-1792.

[15] Pommaret, J.F., Systems of Partial Differential Equations and Lie Pseudogroups, Gordon and Breach Science Publishers, New York, 1978.

[16] Seiler, W.M., Involution: The Formal Theory of Differential Equations and its Applications in Computer Algebra, Algorithms and Computation in Mathematics, vol. 24, Springer-Verlag, New York, 2010.

[17] Singer, I., and Sternberg, S., The infinite groups of Lie and Cartan I. The transitive groups, J. d'Analyse Math. 15 (1965) 1-115.

[18] Stormark, O., Lie's Structural Approach to PDE Systems, Encyclopedia of Mathematics and its Applications, Vol. 80, Cambridge University Press, Cambridge, 2000.

[19] Vessiot, E., Sur la théorie des groupes continues, Ann. École Norm. Sup., 20 (1903) $411-451$.

[20] Weil, A., Oeuvres Scientifiques, vol. 1, Springer-Verlag, New York, 1979. 